\title{
Stiftung für die berufliche Vorsorge der Leitenden Spitalärzte der Schweiz
}

\section{Die Vorsorgeeinrichtung für Leitende Spitalärzte und Chefärzte der Schweiz: Endlich für alle möglich!}

\author{
W. Schweizer
}

PD Dr. med. Walter Schweizer

Kantonsspital

Abteilung Chirurgie

8208 Schaffhausen
Korrespondenz:

Der VLSS (Verein der Leitenden Spitalärzte der Schweiz) und seine Vorsorgestiftung empfehlen Ihnen einen Beitritt zur Stiftung für die berufliche Vorsorge der Leitenden Spitalärzte für das Jahr 2003:

- Während es bis jetzt für alle Leitenden Spitalärzte möglich war, die als selbständig geltenden erworbenen ambulanten Privathonorare auch als angestellte Leitende Spitalärzte und Chefärzte steuerabzugsfähig in einer Pensionskasse zu versichern, war es nur in jenen Kantonen zulässig, auch die Einkünfte von stationären Patienten steuerabzugsfähig in einer Pensionskasse zu versichern, wo diese Honorare als selbständigerworben gegolten haben (siehe unten).

- Wir haben nun von der Vorsorgestiftung aus eine neue Versicherungsmöglichkeit geschaffen, welche es erlaubt, in Kantonen, wo die stationären Einkünfte von Privatpatienten als unselbständiges Einkommen zählen, einen Anschlussvertrag mit der Vorsorgeeinrichtung VLSS spitalweise gemeinsam abzuschliessen. Dies ist seit diesem Sommer rückwirkend per 1. Januar 2003 nun in der ganzen Schweiz möglich. Die Stiftungsaufsicht des Kantons Bern hat unsere Vorsorgereglemente entsprechend genehmigt.

Mit dem Beitritt zur Vorsorgestiftung VLSS (2. Säule) können Sie für das laufende Jahr Ihre Privateinkünfte versichern und die Pensionskas-

Tabelle 1

Auswirkung der Versicherung des Privateinkommens innerhalb der beruflichen Vorsorge (2. Säule) auf die Steuerbelastung am Beispiel des Kantons Bern.

\begin{tabular}{|c|c|c|c|c|c|c|}
\hline Annahme: & \multicolumn{6}{|c|}{$\begin{array}{l}\text { verheirateter Arzt, reformiert; } \\
\text { steuerbares Einkommen aus unselbständiger Erwerbstätigkeit (Grundlohn): } \\
\text { Fr. } 150 \text { 000.-; }\end{array}$} \\
\hline \multicolumn{7}{|c|}{ Beispiel Bern } \\
\hline \multicolumn{2}{|c|}{ Privateinkommen } & $50000 .-$ & $100000 .-$ & $150000 .-$ & $200000 .-$ & $250000 .-$ \\
\hline \multicolumn{2}{|c|}{ Einzahlung in VLSS-Stiftung } & $10000 .-$ & $20000 .-$ & $30000 .-$ & $40000 .-$ & $50000 .-$ \\
\hline \multicolumn{2}{|c|}{ Steuerersparnis pro Jahr } & $4200 .-$ & $8500 .-$ & $19500 .-$ & 22 000.- & $26500 .-$ \\
\hline
\end{tabular}

senbeiträge bei der Einkommenssteuer in Abzug bringen. Dies gilt auch für allfällige Einkaufssummen rückwirkend bis zum 25. Altersjahr.

Die steuerliche Begünstigung macht die 2. Säule gegenüber der Privatvorsorge (Säule 3b) sehr attraktiv. Allfällige Zinsdifferenzen werden durch fehlende Vermittlungsgebühren und durch Steuerabzugsfähigkeit der Beiträge im Rahmen der 2. Säule mehr als kompensiert. Auch die derzeit nicht sehr günstige Lage auf den Aktienmärkten, die uns Zinsnullrunden oder sogar Negativzinsen beschert, wird durch die Möglichkeit der Steuerabzugsfähigkeit mehr als kompensiert. Ein Beispiel der steuerlichen Auswirkung für den Kanton Bern haben wir in Tabelle 1 aufgeführt.

Die Einkünfte aus der ambulanten Sprechstundentätigkeit gelten in allen Kantonen als selbständig erworbene Privateinkünfte und können in jedem Fall bei der Vorsorgestiftung VLSS steuerabzugsfähig mit Einzelanschlussvertrag versichert werden. Die Einkünfte von stationären Privatpatienten können nur in denjenigen Kantonen steuerabzugsfähig in der 2. Säule pensionskassenversichert werden, wo sie AHV-rechtlich als «selbständig erworben» gelten (siehe unten). In den meisten Kantonen aber gelten aufgrund eines Entscheides des Schweizerischen Versicherungsgerichtes die Einkünfte von stationären Privatpatienten als «unselbständig erworben», so dass sie bisher nicht steuerabzugsfähig in einer Pensionskasse versichert werden konnten. In diesen Fällen ist es nun seit neuem möglich, für Kollektive von Spitalärzten aus einem bestimmten Spital nach Absprache mit der Spitalverwaltung unserer Vorsorgestiftung beizutreten, wobei die einzige Voraussetzung die Mitgliedschaft des einzelnen Spitalarztes beim VLSS ist, da unsere berufliche Vorsorgestiftung eine Stiftung des VLSS ist.

Wir sind überzeugt, dass alle Gründe für einen Eintritt in die berufseigene Vorsorgestiftung grundsätzlich auf der Hand liegen, da wir Ihnen ermöglichen, im Rahmen Ihrer Vorsorge erhebliche Einsparungen zu machen und auch die Privateinkünfte wie andere Berufsgattungen längerfristig in einer angemessenen Altersvorsorge zu versichern. Im Artikel 113 der Schweizerischen Bundesverfassung heisst es nämlich: 


\section{Berufliche Vorsorge}

1. Der Bund erlässt Vorschriften über die berufliche Vorsorge.

2. Er beachtet dabei folgende Grundsätze:

a) Die berufliche Vorsorge ermöglicht zusammen mit der Alters-, Hinterlassenenund Invalidenversicherung die Fortsetzung der gewohnten Lebenshaltung in angemessener Weise.

b) Die berufliche Vorsorge ist für Arbeitnehmerinnen und Arbeitnehmer obligatorisch; das Gesetz kann Ausnahmen vorsehen.

c) Die Arbeitgeberinnen und Arbeitgeber versichern ihre Arbeitnehmerinnen und Arbeitnehmer bei einer Vorsorgeeinrichtung; soweit erforderlich, ermöglicht ihnen der Bund, die Arbeitnehmerinnen und Arbeitnehmer in einer eidgenössischen Vorsorgeeinrichtung zu versichern.

d) Selbständigerwerbende können sich freiwillig bei einer Vorsorgeeinrichtung versichern.

e) Für bestimmte Gruppen von Selbständigerwerbenden kann der Bund die berufliche Vorsorge allgemein oder für einzelne Risiken obligatorisch erklären.

3. Die berufliche Vorsorge wird durch die Beiträge der Versicherten finanziert, wobei die Arbeitgeberinnen und Arbeitgeber mindestens die Hälfte der Beiträge ihrer Arbeitnehmerinnen und Arbeitnehmer bezahlen.

4. Vorsorgeeinrichtungen müssen den bundesrechtlichen Mindestanforderungen genügen; der Bund kann für die Lösung besonderer Aufgaben gesamtschweizerische Massnahmen vorsehen.

Bei den Leitenden Spitalärzten und Chefärzten wurde der Verfassung der Eidgenossenschaft in vielen Kantonen bisher keine Beachtung geschenkt, da die Altersvorsorge für stationäre Privateinkünfte nicht im Rahmen der beruflichen Vorsorge versicherungsfähig war.

Wenn Sie selbst oder die Leitenden und Chefärzte Ihres Spitals am Eintritt in diese berufliche Vorsorgestiftung des VLSS interessiert sind, dann setzen Sie sich mit einem unserer folgenden Berater oder direkt mit unserer Geschäftsstelle Pendia Associates AG in Zürich in Verbindung:

- Ärzteberatungsstelle ABC Bühler und Partner AG, Auf Weinbergli 16, 6005 Luzern, Tel. 041 36850 40;

- Roger Clénin, Monbijoustrasse 10, Postfach 8718, 3001 Bern, Tel. 03138051 63;
- Willi Horndasch, Engimattstrasse 22, 8002 Zürich, Tel. 0120176 79;

- P. A. Steiner, Avenue du Theâtre 1, 1005 Lausanne, Tel. 02134505 05;

- Lotti Gautschi und Sabine Nussberger, Pendia Associates AG Zürich, Tel. 0128920 89, E-Mail: lotti.gautschi@pendia.ch.

Diese Berater sind gerne bereit, Sie auch auf dem möglicherweise beschwerlichen Weg zusammen mit der Spitalverwaltung zu beraten, um diese Versicherung möglichst rasch bei den Steuerbehörden deklarationsfähig zu machen. Natürlich müssen die Spitalärzte einzelner Kantone für den $\mathrm{zu}$ erwartenden und $\mathrm{zu}$ erreichenden Steueroptimierungsgewinn die entsprechenden Erkundigungen und Verhandlungen mit der Spitalverwaltung und den jeweils zuständigen Steuerbehörden selber führen respektive zusammen mit den Beratern oder allenfalls Anwälten.

Kantone, in welchen die Mitglieder die einbezahlten Beiträge aus stationären Behandlungen steuermässig nicht in Abzug bringen konnten und wo dies nun mit dem neuen «Vorsorgereglement für leitende Spitalärzte von angeschlossenen Spitälern» spitalweise möglich ist:

- Aargau, Appenzell Ausserrhoden/Innerrhoden, Luzern, Schaffhausen, St. Gallen, Zug, Zürich.

Kantone, bei welchen es für die Mitglieder bei den einbezahlten Beiträgen aus stationären und ambulanten Behandlungen bis heute keine steuerlichen Probleme gegeben hat:

- Basel-Landschaft/-Stadt, Bern, Freiburg, Glarus, Jura, Neuenburg, Nidwalden, Obwalden, Schwyz, Solothurn, Thurgau, Uri.

Auch in diesen Kantonen ist aber selbstverständlich ein spitalweiser Anschluss an die Vorsorgeeinrichtung VLSS möglich.

Kantone, welche andere Varianten ermöglichen, bei denen aber ebenfalls ein Anschluss an die Vorsorgeeinrichtung VLSS möglich ist:

- Genf, Lausanne, Tessin.

Zum Schluss bleibt zu erwähnen, dass der Stiftungsrat dieser Stiftung unentgeltlich und ehrenamtlich tätig ist und dass Ihnen die von Ihnen einbezahlten Beiträge und die Erträge vollumfänglich gutgeschrieben werden. Davon ausgenommen sind die üblichen Verwaltungsgebühren, die wir so niedrig halten wie möglich. 